\title{
NON-CONTRAST MR LYMPHOGRAPHY OF RARE LYMPHATIC ABNORMALITIES
}

\author{
M.D. Seckeler, L. Moedano, D. Mustacich, B.T. Kalb, \\ M. Saranathan, J.-P. Galons, M.H. Witte
}

Departments of Pediatrics (Cardiology) (MDS), Family Medicine (LM), Surgery (DM,MHW), and Medical Imaging (BTK,MS,J-PG), University of Arizona, Tucson, Arizona, USA

\section{ABSTRACT}

Detailed imaging of the lymphatic system often requires direct injection of contrast into lymph nodes which can be technically challenging, time consuming, and produce painful stimuli. We sought to describe the use of non-contrast MR lymphography (NCMRL) for normal controls and patients with a variety of rare disorders associated with lymphatic pathologies. Two control subjects and five affected patients (decompensated Fontan circulation, central conducting lymphatic abnormality, familial lymphedema and two with Gorham-Stout disease) were studied. NCMRL images were segmented in a semiautomated fashion and the major lymphatic channels and thoracic duct (TD) highlighted for identification. Adequate imaging was available for both controls and 4/5 affected patients; the youngest patient could not be imaged given patient motion. For the two controls, the TD was seen in the expected anatomic location. For the decompensated Fontan patient, there were numerous tortuous lymphatic channels, predominantly in the upper chest and neck. For the familial lymphedema patient, a TD was not identified; instead, peripheral lymphatic collaterals near the lateral chest walls. For the first GorhamStout patient, the TD was enlarged with large intrathoracic lymph collections. For the second Gorham-Stout patient, there were bilateral TD with lymph collections in vertebral bodies. Using NCMRL, we were able to image normal and abnormal lymphatic systems. An important learning point is the potential need for sedation for younger patients due to long image acquisition times and fine resolution of the structures of interest.

Keywords: non-contrast MR lymphography, thoracic duct, Gorham-Stout syndrome, multiple lymphangiomatosis, congenital lymphedema, Fontan procedure, chylous reflux

With improvements in non-invasive imaging techniques, the "invisible" lymphatic circulation is finally becoming easier to see. In particular, the use of dynamic contrast MR lymphography has been used to guide percutaneous lymphatic interventions $(1,2)$. However, this technique is limited by its more invasive approach which requires direct needle access of lymph nodes to allow for gadolinium infusion as well as challenges in maintaining the needle position in the lymph node during imaging (3-5). Non-contrast MR lymphography (NCMRL) is an attractive, completely non-invasive alternative to image the lymphatic system which has mostly been utilized in cases of lymphedema (6-11) or for pre-procedure planning (12). We hypothesized that NCMRL could be effectively used to image the central lymphatics and thoracic 
duct (TD) in a variety of rare disorders associated with central lymphatic abnormalities.

\section{MATERIALS AND METHODS}

Two control subjects (under a University of Arizona Institutional Review Boardapproved protocol) and five patients with serious medical conditions associated with lymphatic abnormalities [decompensated Fontan circulation, central conducting lymphatic abnormality, familial lymphedema and two with Gorham-Stout disease (multiple lymphangiomatosis with bone involvement)] were studied. Using a 3T Siemens Skyra MRI scanner running E11 software (Siemens Medical Solutions USA, Inc, Malvern, PA), coronal images of the chest or chest/abdomen were acquired using 3D HASTE sequences. Sequence parameters: TR: $5000 \mathrm{~ms}$, TE 701 ms, Echo Train length 182, Flip Angle 150, FOV 260x 440, Slice Thickness: $2.2 \mathrm{~mm}, 64$ slices, Average: 1; Acquisition Time: $3.36 \mathrm{~min}$. For chest imaging an additional set of coronal images were collected using a gated-3DSPACE pulse sequence using the following parameters: TR: $8400 \mathrm{~ms}$, TE $700 \mathrm{~ms}$, Echo Train Length: 180, Flip Angle 115, FOV: 400 x 400; Slice Thickness: $1.1 \mathrm{~mm}, 80$ slices, Average: 2; Acquisition Time: 13 min. Images were segmented in a semi-automated fashion with Philips IntelliSpace Portal (Philips Medical Systems, The Netherlands) and the major lymphatic channels and TD highlighted in green to aid in identification.

\section{RESULTS}

Adequate NCMRL imaging was available for two controls and 4/5 patients with lymphatic abnormalities [median age 20.6 years (range 11.1 to 39.9 years)]; the youngest patient who suffered from central conducting lymphatic abnormality was not sedated and could not hold still to allow for adequate imaging. A composite of the imaging of the two controls and four patients is shown in Fig. 1. For the two controls, the TD was identified and in the expected anatomic location (Fig. $1 A, B)$. For the decompensated Fontan patient (Fig. 1C), there were numerous tortuous lymphatic channels identified; there was also an area of small bowel that correlated with an area of increased radiotracer uptake on SPECT/CT (Fig. 1C, arrowheads). For the familial lymphedema patient (Fig. 1D), no TD was identified, only numerous peripheral lymphatic collaterals and a fluid collection posterior to the spine (Fig. 1D, arrow). For the first Gorham-Stout patient (Fig. 1E), the TD appeared enlarged with large intrathoracic lymph collections. For the second GorhamStout patient (Fig. $1 F$ and Fig. 2), there were bilateral TD with two lymph collections within adjacent vertebral bodies that correlated with areas of increased radiotracer uptake on SPECT/CT (Fig. 2, arrow). There were no complications from imaging.

\section{DISCUSSION}

This is the first case series to report imaging from non-contrast MR lymphography of the central lymphatics and thoracic duct for a variety of rare conditions with associated central lymphatic abnormalities. These findings provide support for the incorporation of this technique in the armamentarium of non-invasive lymphatic imaging which already includes lymphoscintigraphy and SPECT/CT (13).

Each of these cases highlights the value of NCMRL in screening, diagnosing and understanding lymphatic disorders. For the two controls (Fig. 1A,B), we were able to identify a normal TD, whereas prior reports only identified a damaged, leaking TD (8) or the cisterna chyli using non-contrast MR scans $(14,15)$. This was useful to establish the validity of our scanning technique. Recently in pediatric patients with complex congenital heart disease, whole-body lymphangioscintigraphy and SPECT/CT have suggested central lymphatic obstruction (16). Our findings confirmed this in a patient with decompensated Fontan physiology (Fig. 1C). 


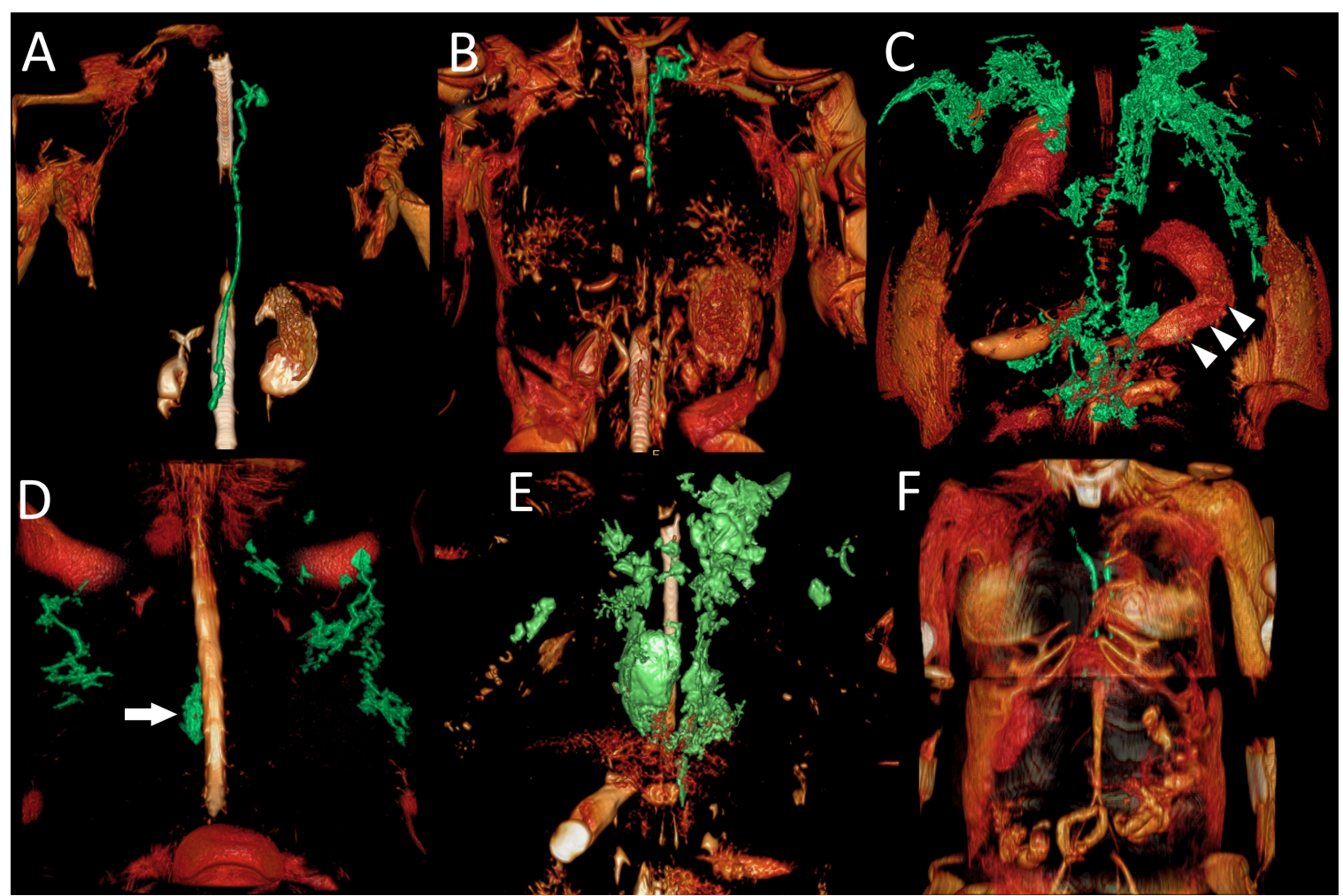

Fig. 1. Non-contrast MR lymphangiograms from controls and patients. $(A, B)$ The two control subjects show the thoracic duct (green) in the expected location, including the region of the jugulo-subclavian junction. (C) The patient with decompensated Fontan physiology showing numerous, dilated, tortuous lymphatic collaterals, particularly in the cervical region. In addition, the area of small intestine of suspected protein leak is identified by the arrowheads. D) The patient with familial lymphedema shows no TD with a small fluid collection posterior to the spine (arrow) and prominent bilateral collaterals along the chest wall. E) The first Gorham-Stout patient shows an enlarged TD with large intrathoracic lymph collections. F) The second Gorham-Stout patient shows bilateral $T D$. The lymph collections in the vertebral bodies are not seen in this projection.

We were able to not only identify numerous dilated cervical lymphatic collaterals, but also an area of small bowel that correlated with an area of increased tracer uptake on SPECT/CT. This patient suffered from protein losing enteropathy, and we suspected from a prior lymphangioscintigram and SPECT/CT the jejunal region was the source intestinal loss of lymph fluid. Unfortunately, the patient was too frail and immunocompromised to undergo an attempt at surgical resection of the affected bowel to confirm this. For the familial lymphedema patient (Fig. 1D), the absence of the TD with prominent bilateral collaterals along the chest wall were not expected based on his clinical findings or prior lymphatic imaging. This provided novel insight into his underlying pathology and the etiology of his lymphedema. Imaging of other affected family members is anticipated in the future to see if they have similar findings. For the first Gorham-Stout patient (Fig. 1E), the dilated TD had not been appreciated using other lymphatic imaging modalities. For the second Gorham-Stout patient (Figs. 1F,2), identification of bilateral TD was an interesting normal variant. However, more compelling and insightful into the pathology of Gorham-Stout was identifiable lymph accumulation in bones before osteoclastic 


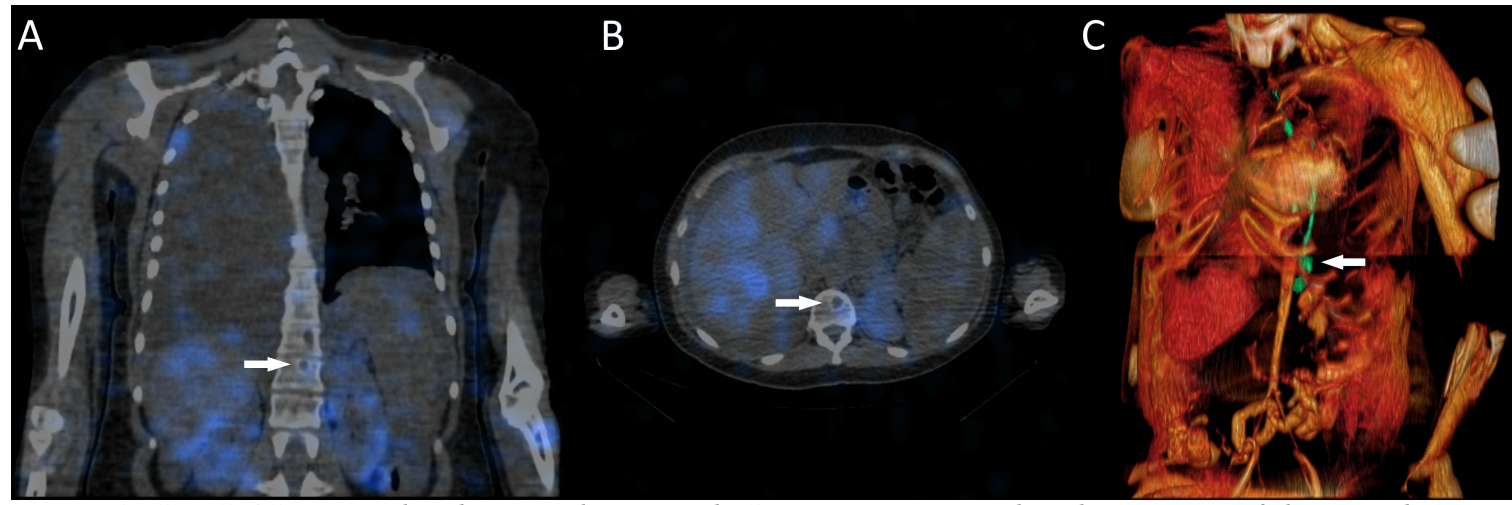

Fig. 2. (A) SPECT/CT coronal and (B) axial views and (C) non-contrast MR lymphangiogram of the second Gorham-Stout patient. A and B) The arrow shows the area of increased radiotracer uptake on SPECT/CT in the vertebral body in the same area of the lytic bone lesions, suggestive of a lymph collection. B) Non-contrast MR lymphangiogram confirms two lymph collections in the same area (arrow).

destruction as suggested on prior lymphangioscintigraphy and SPECT/CT, lending support to the "lymph in bone" etiology of the disease.

This study is limited by the small number of subjects, and expansion of this technique to additional sites in the future will help demonstrate the utility of NCMRL. The image acquisition was safe for all subjects, but an important learning point is the potential need for sedation or anesthesia for some younger patients due to the long image acquisition times and fine resolution of the structures of interest. Using non-contrast MR lymphography, we were able to image the central lymphatics in normal subjects and patients with a variety of disorders associated with lymphatic involvement. The use of this technique in conjunction with lymphoscintigraphy and SPECT/CT provides additional insight into the anatomic and physiologic mechanisms of a variety of complex lymphatic disorders and should guide future therapies, both surgical and interventional.

\section{ACKNOWLEDGMENTS}

This work was supported by funded from the intramural Translational Imaging Program Projects Stimulus Funds (MHW) and National Heart, Lung and Blood
Institute R25HL108837 (LM).

\section{CONFLICT OF INTEREST AND DISCLOSURE}

The authors declare no competing financial interests exist.

\section{REFERENCES}

1. Dori, Y, MS Keller, JJ Rome, et al: Percutaneous lymphatic embolization of abnormal pulmonary lymphatic flow as treatment of plastic bronchitis in patients with congenital heart disease. Circulation 133 (2016), 1160-1170.

2. Itkin, MG, FX McCormack, Y Dori:

Diagnosis and treatment of lymphatic plastic bronchitis in adults using advanced lymphatic imaging and percutaneous embolization. Ann. Am. Thorac. Soc. 13 (2016), 1689-1696.

3. Nadolski, GJ, M Itkin: Feasibility of ultrasound-guided intranodal lymphangiogram for thoracic duct embolization. J. Vasc. Interv. Radiol. 23 (2012), 613-616.

4. Itkin, M, GJ Nadolski: Modern techniques of lymphangiography and interventions: Current status and future development. Cardiovasc. Intervent. Radiol. 41 (2018), 366376.

5. Chick, JFB, GJ Nadolski, AR Lanfranco, et 
al: Dynamic contrast-enhanced magnetic resonance lymphangiography and percutaneous lymphatic embolization for the diagnosis and treatment of recurrent chyloptysis. J. Vasc. Interv. Radiol. 30 (2019), 1135-1139.

6. Takahashi, H, S Kuboyama, H Abe, et al: Clinical feasibility of noncontrast-enhanced magnetic resonance lymphography of the thoracic duct. Chest 124 (2003), 2136-2142.

7. Arrivé, L, S Derhy, S El Mouhadi, et al: Noncontrast magnetic resonance lymphography. J. Reconstr. Microsurg. 32 (2016), 80-86.

8. Kim, EY, HS Hwang, HY Lee, et al: Anatomic and functional evaluation of central lymphatics with noninvasive magnetic resonance lymphangiography. Medicine (Baltimore) 95 (2016), e3109.

9. Arrivé, L, S Derhy, B Dahan, et al: Primary lower limb lymphoedema: Classification with non-contrast MR lymphography. Eur. Radiol. 28 (2018), 291-300.

10. Cellina, M, G Oliva, A Menozzi, et al: Noncontrast magnetic resonance lymphangiography: an emerging technique for the study of lymphedema. Clin. Imaging 53 (2019), 126-133.

11. Cellina, M, D Gibelli, C Floridi, et al: Volumetric analysis of non-contrast magnetic resonance lymphangiography in patients affected by lower extremities primary lymphedema. Radiol. Med. 125 (2020), 432-435. doi: 10.1007/s11547-019-01122-9. Online ahead of print.

12. Pamarthi, V, WM Pabon-Ramos, V Marnell, LM Hurwitz: MRI of the central lymphatic system: Indications, imaging technique, and pre-procedural planning. Top. Magn. Reson. Imaging 26 (2017), 175-180. doi:10.1097/RMR.0000000000000130
13. The diagnosis and treatment of peripheral lymphedema: 2020 Consensus Document of the International Society of Lymphology. Lymphology 53 (2020), 3-19.

14. Verma, SK, DG Mitchell, D Bergin, et al: Dilated cisternae chyli: A sign of uncompensated cirrhosis at MR imaging. Abdom. Imaging 34 (2009), 211-216.

15. Ito, K, A Shimizu, M Tanabe, et al: Cisterna chyli in patients with portal hypertension: evaluation with MR imaging. J. Magn. Reson. Imaging 35 (2012), 624-628.

16. Kuo, PH, BJ Barber, RI Kylat, et al: Wholebody lymphangioscintigraphy and SPECT/CT in children with lymphatic complications after surgery for complex congenital heart disease. Lymphology 52 (2019), 157-165.

\author{
Michael D. Seckeler, MD, Msc \\ Department of Pediatrics (Cardiology) \\ University of Arizona \\ 1501 N. Campbell Avenue \\ PO Box 245073 \\ Tucson, AZ 85724-5073 \\ Phone (520) 626-5585 \\ Fax (520) 626-6571 \\ E-mail: mseckeler@peds.arizona.edu
}

